\title{
ESTIMATING THE NUMBER OF COMPONENTS IN AN OSL DECAY CURVE USING THE BAYESIAN INFORMATION CRITERION
}

\author{
JUN PENG ${ }^{1}$, ZHIBAO DONG ${ }^{1}$, FENGQING HAN $^{2}$, YUANHONG HAN $^{\mathbf{3}}$ and XUELING DAI ${ }^{\mathbf{1}}$ \\ ${ }^{1}$ Cold and Arid Regions Environmental and Engineering Research Institute, Chinese Academy of Sciences, Lanzhou 730000, China \\ ${ }^{2}$ Qinghai Institute of Salt Lakes, Chinese Academy of Sciences, Xining 810008, China \\ ${ }^{3}$ Lanzhou Center for Oil and Gas Resources, Institute of Geology and Geophysics, CAS, Lanzhou 730000, China
}

\begin{abstract}
The optically stimulated luminescence (OSL) decay curve is assumed to consist of a number of first-order exponential components. Improper estimation of the number of components leads to under- or over-fitting of the curve under consideration. Hence, correct estimation of the number of components is important to accurately analyze an OSL decay curve. In this study, we investigated the possibility of using the Bayesian Information Criterion to estimate the optimal number of components in an OSL decay curve. We tested the reliability of this method using several hundred measured decay curves and three simulation scenarios. Our results demonstrate that the quality of the identification can be influenced by several factors: the measurement time and the number of channels; the variability of the decay constants; and the signal-to-noise ratios of a decaying component. The results also suggest that the Bayesian Information Criterion has great potential to estimate the number of components in an OSL decay curve with a moderate to high signal-to-noise ratio.
\end{abstract}

Keywords: OSL decay curves, number of components, Bayesian Information Criterion.

\section{INTRODUCTION}

Optically stimulated luminescence (OSL) decay curves are fitted as the sum of a number of first-order exponential decaying components (Adamiec, 2005; Bluszcz and Adamiec, 2006; Li and Li, 2006a, 2006b). Many researchers have estimated the number of components in an OSL decay curve empirically ( $\mathrm{Li}$ and $\mathrm{Li}$, 2006b; Peng and Han, 2013). However, the number of components varies significantly for natural sediments (Jain et al., 2003; Singarayer and Bailey, 2003; Tokuyasu et al., 2010). Fitting a decay curve with more components would improve the quality of the fit, but may give rise to the problem of over-fitting. In contrast, under-fitting may

Corresponding author: J. Peng

e-mail: pengjun10@mails.ucas.ac.cn occur if the curve is fitted with fewer components than is really needed. Because of the importance of these curves for determining the luminescence properties of sediment deposits, it is important to determine whether an objective method can be used to estimate the required number of components. This is particularly significant for fastcomponent OSL dating. Checking the uncertainties of the fitted parameters is a simple and direct method for diagnosing over-fitting. A Hessian matrix with a very large condition number means that the model is ill-conditioned and that this would lead to high uncertainty of the estimate. However, this simple method does not detect under-fitting. Adamiec (2005) identified the number of decaying components using the $F$-statistic. Based on this approach, Bluszcz and Adamiec (2006) proposed a method that employs a similar but more general protocol and 
that aims to find a global minimum for the problem under consideration.

From a statistical perspective, estimating the optimal number of components is a model selections problem (Sivia, 1996). The Bayesian Information Criterion is a powerful tool that can be used to tackle such a problem (Schwarz, 1978), since it correlates the likelihood function for a model with the relevant number of parameters. The Bayesian Information Criterion has been applied to choose a reasonable number of parameters in the analysis of statistical age models (Galbraith, 1988; Galbraith et al., 1999; Galbraith and Roberts, 2012). However, when it comes to analysis of an OSL decay curve, it may be difficult to apply this approach due to at least three problems: (1) fitting an OSL decay curve with a number of first-order exponential components is a notoriously illconditioned problem; (2) the luminescence properties (e.g., variations in OSL intensity and decay constants) of a decay curve of natural sediments behave differently from place to place, which further complicates the problem; and (3) the uncertainty that results from counting statistics would unavoidably degrade the quality of the data and decrease the accuracy of the estimate. In the present study, we investigated the feasibility of estimating the number of components in an OSL decay curve based on the Bayesian Information Criterion. We tested the applicability of this method using 624 measured decay curves with variable OSL intensities. We also simulated several simple scenarios to explore the influences of the data resolution, the OSL intensities, and decay rates on identifying the optimal number of components.

\section{METHODS}

For simplicity, we consider only the continuous wave (CW) type of OSL decay curve, although the same methodology would apply to the linear modulation (LM) type of OSL decay curve. If we assume that the ideal OSL signals can be expressed as:

$$
I\left(x_{i},\left[A_{j}\right],\left[B_{j}\right], k\right)=\sum_{j=1}^{k} A_{j} B_{j} e^{\left(-B_{j} x_{i}\right)}
$$

where $x_{i}$ represents the $i$-th time value (i=1 to $\left.\mathrm{n}\right)$, and $\left[A_{\mathrm{j}}\right]$ and $\left[B_{\mathrm{j}}\right]$ denote the number of trapped electrons and the decay constant, respectively, for each of the $j=1$ to $k$ components. If we further assume that photon counts follow a Poisson distribution:

$$
y_{i} \sim \pi\left(\overline{y_{i}}\right)
$$

where $y_{\mathrm{i}}$ is the $i$-th observed number of counts and the real value of $y_{\mathrm{i}}$ is denoted by $\overline{y_{i}}$. The likelihood function for the relevant parameters would then be:

$$
\begin{aligned}
& p\left\{y_{i} / I\left(x_{i},\left[A_{j}\right],\left[B_{j}\right], k\right)\right\}= \\
& \frac{I\left(x_{i},\left[A_{j}\right]\left[B_{j}\right], k\right)^{y_{i}} e^{-I\left(x_{i},\left[A_{j}\right]\left[B_{j}\right], k\right)}}{y_{i} !}
\end{aligned}
$$

where / means "given" (i.e., a conditional probability), and $p\{X\}$ means "the probability of $X$ ". If we further suppose that each $y_{\mathrm{i}}$ is independent of the others, then the joint-likelihood function can be described as:

$p\left\{\left[y_{i}\right] I\left(\left[x_{i}\right],\left[A_{j}\right],\left[B_{j}\right], k\right)\right\}=$
$\prod_{i=1}^{n} \frac{I\left(x_{i},\left[A_{j}\right],\left[B_{j}\right], k\right)^{y_{i}} e^{-I\left(x_{i},\left[A_{j}\right],\left[B_{j}\right], k\right)}}{y_{i} !}$

Combining Eq. 2.4 with Eq. 2.1, we can rewrite Eq. 2.4 as follows:

$$
\begin{aligned}
& p\left\{\left[y_{i}\right] /\left[x_{i}\right],\left[A_{j}\right],\left[B_{j}\right], k\right\}= \\
& \prod_{i=1}^{n} \frac{\left(\sum_{j=1}^{k} A_{j} B_{j} e^{\left(-B_{j} x_{i}\right)}\right)^{y_{i}} \cdot e^{-\sum_{j=1}^{k} A_{j} B_{j} e^{\left(-B_{j} x_{i}\right)}}}{y_{i} !}
\end{aligned}
$$

If $k$ is known in Eq. 2.5, then the optimal estimate can be obtained through a maximum-likelihood estimation method. The number of components $(k)$ can therefore be estimated using the Bayesian Information Criterion. Schwarz (1978) defines the Bayesian Information Criterion $(\mathrm{BIC})$ as:

$$
B I C=-2 L_{\max }+N_{p} \log (n)
$$

where $L_{\max }$ denotes the maximized log-likelihood function value, $N_{\mathrm{p}}$ is the number of parameters, and $n$ is the number of observations. Combining Eq. 2.5 and 2.6 leads to:

$B I C(k)=-2 \sum_{i=1}^{n} \ln \frac{\left(\sum_{j=1}^{k} \overline{A_{j}} \overline{B_{j}} e^{\left(-\overline{B_{j}} x_{i}\right)}\right)^{y_{i}} \cdot e^{-\sum_{j=1}^{k} \overline{A_{j} \bar{B}_{j}}\left(-\overline{B_{j} x_{i}}\right)}}{y_{i} !}+2 k \ln (n)$

where $\overline{A_{j}}, \overline{B_{j}}(j=1,2, \ldots, k)$ are parameters that maximize the value of Eq. 2.5 for a given $k$. Note that the coefficient of the second term on the right side of Eq. 2.7 needs to be modified to $2 k+1$ if a constant component is added to account for background subtraction. The maximumlikelihood estimation of Eq. 2.5 can be approximated using a weighted nonlinear least-squares method if we assume that the number of photons follows a normal distribution with a mean equal to the number of counts and a standard deviation equal to the square root of the number of counts (Bluszcz and Adamiec, 2006). In all of the analyses presented in this study, we performed weighted nonlinear least-squares fitting using a modified version of the decompc() function, which allows a constant component to be subtracted, in the numOSL (version 
1.1) package (Peng et al., 2013) for the R statistical software, and the optimal number of components can be estimated using the BIC from integers in the interval [1, 5]. We automatically initialized the parameters using a differential evolution method (Storn and Price, 1997), as suggested by Bluszcz and Adamiec (2006). Bearing in mind the possibility of bad performance of the considered model, application of the BIC was not direct (i.e., the condition of the model was also considered as a test of the quality of the fit). This was done based on the fact that the BIC does not consider the property of the model under consideration while fitting the OSL decay curve as an "ill-posed" problem. The general procedure can be described as finding the $k$ value that gives the minimum BIC value from among the numbers of components that remain the model non-singular. The approach consists of the following steps:

1) Set the number of components to $k=1$ and fit the decay curve with the weighted nonlinear leastsquares method, then calculate $\operatorname{BIC}(k)$.

2) Fit the decay curve with $k+1$ components and test whether the model is nearly singular. If it is nonsingular, continue with step 3 ; if it is singular, continue with step 4.

3) Calculate $\mathrm{BIC}(k+1)$. If $\mathrm{BIC}(k+1)<\mathrm{BIC}(k)$, repeat step 2; if not, continue with step 4 .

4) Output the estimated number of components $(k)$ and associated parameters and end the analysis.

\section{ESTIMATING THE NUMBER OF COMPO- NENTS USING MEASURED OSL CURVES}

We assessed the estimates produced by the BIC method using measured OSL curves for two sand samples, GL1-1 and GL1-2 (Peng and Han, 2013), that were obtained from the southern edge of the Tengger Desert in northern China. The CW-OSL measurements were conducted using an automated Risø TL/OSL-20 reader. Decay curves were obtained by stimulating a multiple-grain aliquot under a blue $\operatorname{LED}(\lambda=470 \pm 20 \mathrm{~nm})$ source at $130^{\circ} \mathrm{C}$ for $40 \mathrm{~s}$ using 250 equal-width channels. Decay curves that corresponded to 0 regenerative doses were excluded from the analysis because of their low signal-tonoise ratio. We obtained 624 decay curves from 48 aliquots. Fig. 1 shows the distributions of the estimated decay rates $(B)$ and constant $(C)$ for values of $k$ ranging from 1 to 4 .

Table 1 shows that the decay curve with the highest OSL intensity required the most components to optimize the fitting. This is because a dim decay curve has a low signal-to-noise ratio, and hence cannot be fitted with many components, whereas a bright decay curve contains more information and thus needs more components to improve the quality of the fitting (Adamiec, 2005). Because the estimated constants in Fig. 1 are comparable to the background intensities listed in Table 1 (after accounting for their standard deviations), the results are reliable. However, the estimated constants in Fig. 1 were significantly larger than the expected background from a reader. This suggests that the estimated constant should be regarded as the sum of the slower components and the background rather than solely as the background. It also suggests that the measured curves contained one or more slower components and that a stimulation time of $40 \mathrm{~s}$ was not enough to completely eliminate these components (see Section 4 for details).

\section{SIMULATED SCENARIOS}

The potential to identify the optimal number of components can be affected by several factors (Bluszcz and Adamiec, 2006). These factors can be categorized as follows:

1) Controllable factors: These are factors that the researcher can control, and include the stimulation time and the number of channels used in the measurement. The relationship of the decay constants to the total measurement time (Bluszcz and Adamiec, 2006) can strongly affects the correctness of the estimate, as we showed in Section 3. If the mean life of a decaying component is shorter than the measurement time (i.e., its intensity decreases to a negligible level at the end of the stimulation), then it may be isolated correctively from the measured curve. Using a large number of channels improves the quality of the fitting (Steffen et al., 2009) and should also increase the reliability of the identified number of components.

2) Sample-dependent factors: These factors relate to the inherent properties of the sample material, and include the OSL intensities of the decaying components and the difference between decay constants. The quality of the estimate would be decreased for a dim sample that has a low signal-to-noise ratio. Single grains of quartz from natural samples vary significantly in their OSL intensity and sensitivity (Duller et al., 2000; Rhodes, 2007; Duller, 2008; Fitzsim-

Table 1. A summary of the average OSL intensities and the average background intensities for decay curves with $\mathrm{k}$ values ranging from 1 to 4 and a sample size of $\mathrm{N}$. The OSL intensity was calculated using the sum of the counts from the first two channels and the background intensity was calculated using the average counts from the last 20 channels. Note that the calculation of the background intensity differs from that in the Analyst software (Duller, 2007). In order to allow a direct comparison with the estimated constant, the background intensity was not multiplied by the number of integration channels used to calculate the net OSL signal. Values are mean $\pm S D_{\text {mean. }}$.

\begin{tabular}{cccc}
\hline $\boldsymbol{k}$ & $\boldsymbol{N}$ & $\begin{array}{c}\text { OSL intensity } \\
\text { (counts/0.16 s) }\end{array}$ & $\begin{array}{c}\text { Background intensity } \\
\text { (counts/0.16 s) }\end{array}$ \\
\hline 1 & 22 & $775.50 \pm 82.31$ & $111.80 \pm 4.17$ \\
2 & 232 & $1701.28 \pm 87.47$ & $128.57 \pm 1.43$ \\
3 & 345 & $4598.00 \pm 141.64$ & $187.12 \pm 3.64$ \\
4 & 25 & $9497.20 \pm 1338.98$ & $243.82 \pm 14.00$ \\
\hline
\end{tabular}



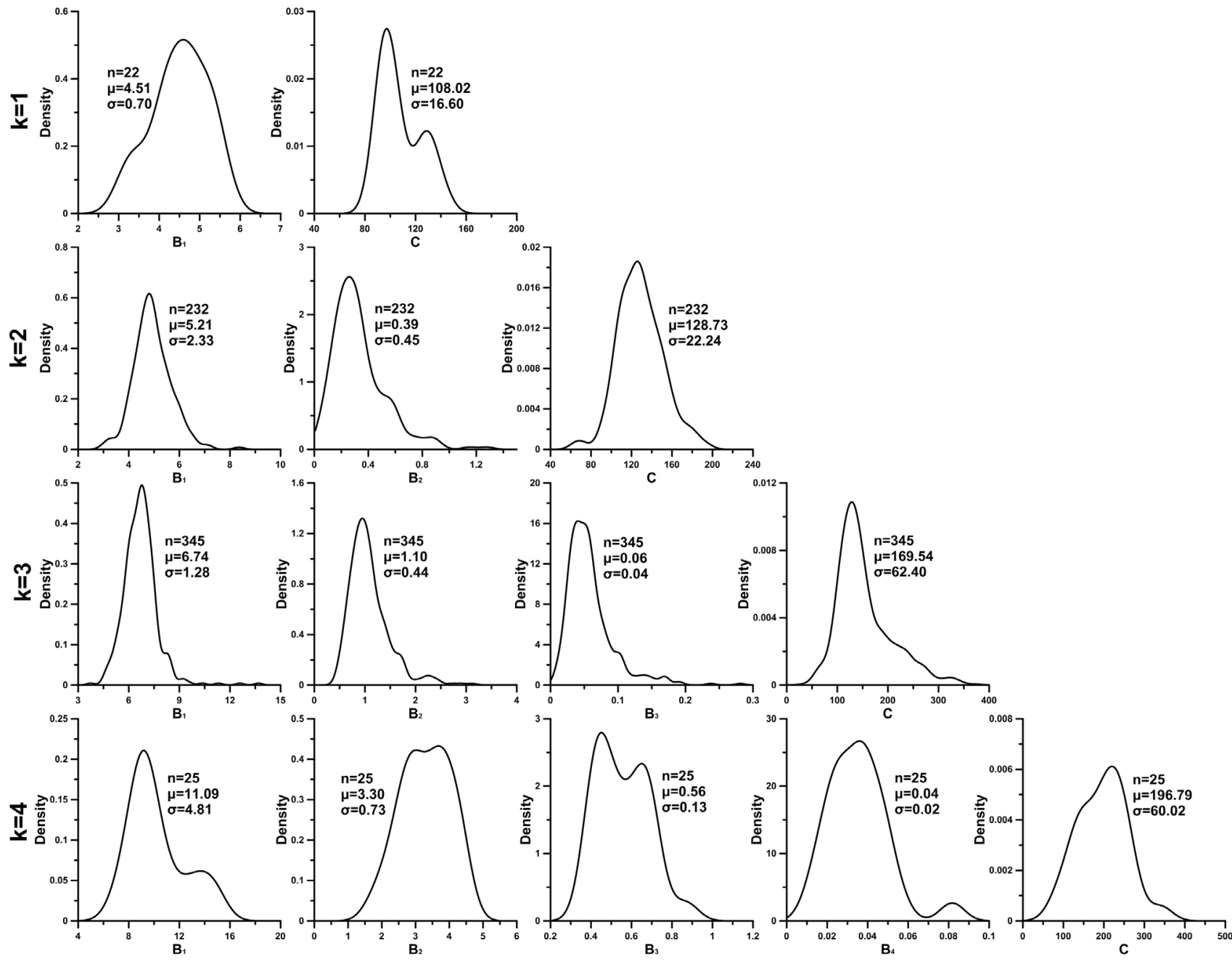

Fig. 1. Distributions of the estimated decay rates $\left(B_{i}\right)$ and constants (C, equivalent to the background intensity) for $\mathrm{k}=1,2,3$, and $4 . \mathrm{n}$ is the number of decay curves, $\mu$ and $\sigma$ denote the average and standard deviation of a decay rate or a constant, respectively. The density plot was smoothed using a Gaussian kernel.

mons, 2011). Variations also exist in OSL intensities between decaying components within a decay curve (Adamiec, 2000). The variation in intensity of a multiple-grain aliquot that consists of a number of quartz grains is more complicated, as it depends on the number of grains and the homogeneity of intensities of the grains (Adamiec, 2005; Rhodes, 2007). Only one component can be isolated when two decay constants are indistinguishable from each other. Fortunately, the decay constants of measured curves from natural samples are usually well distinguished from each other (Jain et al., 2003; Singarayer and Bailey, 2003; Tokuyasu et al., 2010; the present study).

3) The uncertainty that arises from counting statistics. The variance for photon counts is over-dispersed $(\mathrm{Li}$, 2007) to different degrees compared with the assumed Poisson variance, depending on the reader system employed for the measurement (Adamiec et al., 2012). A counting system with a larger degree of over-dispersion (i.e., a big correction factor) would produce noisier data, and the influence of that noise would be especially significant for dim samples.

The cumulative effect of the above mentioned factors on the quality of the estimate can be complicated. For example, Bluszcz and Adamiec (2006) found that four components could be isolated properly only under very special circumstances (i.e., bright decay curves with easily distinguishable decay rates) with a 100 -s measurement and 255 equal-width channels. This means that if the brightness of a decay curve is not sufficiently high or if the decay constants are not sufficiently distant, then a longer stimulated time and more channels would be needed to ensure that the estimate is correct.

To examine these factors, we developed three simple scenarios (each simulated 5,000 times) to assess the influences of the first two kinds of factors on the correctness of the estimate. In Scenario 1, we investigated the possible influences of the measurement time and the 
number of channels on the resultant estimate. In Scenario 2, we investigated the effect of signal intensity (relative to the background level). In Scenario 3, we explored the influence of variation in the decay constants on the result. Decay curves were synthesized with three components $(k=3)$ and were randomized using a Poisson distribution generator. The background was taken to be 40 in all scenarios. The scenarios were simulated using the following parameter values:

Scenario 1: The decay rates were set equal to the estimated average decay constants of the 345 measured decay curves that had an estimated number of components equal to 3 (Fig. 1). The three numbers of trapped electrons were set equal to 1,000 . The number of channels was generated from integers in the interval $[10,300]$ with equal probability. The total measurement time (s) was generated uniformly from the interval $(5,50)$.

Scenario 2: The decay rates were set equal to the estimated average decay constants of the 345 measured decay curves that had an estimated number of compo- nents equal to 3 (the same as in Scenario 1). The numbers of trapped electron was generated uniformly and independently from the space $(100,1000)$. The number of channels and the simulation time were fixed at 250 and $40 \mathrm{~s}$, respectively.

Scenario 3: The decay rates were generated with a truncated normal distribution (all values within $2 \sigma$ of the mean), with the mean values and standard deviations equal to the estimated means and standard deviations of the 345 measured curves that had an estimated number of components equal to 3 . That is, only decay constants that were generated within two standard deviations of the mean were accepted. Note that the lower limit of the smallest decay rate (with an average of 0.06 and a standard deviation of 0.04 ) was forced to zero. The three numbers of trapped electrons were set equal to 1,000 (the same as in Scenario 1). The number of channels and the measurement time were fixed at 250 and $40 \mathrm{~s}$, respectively (the same as in Scenario 2).
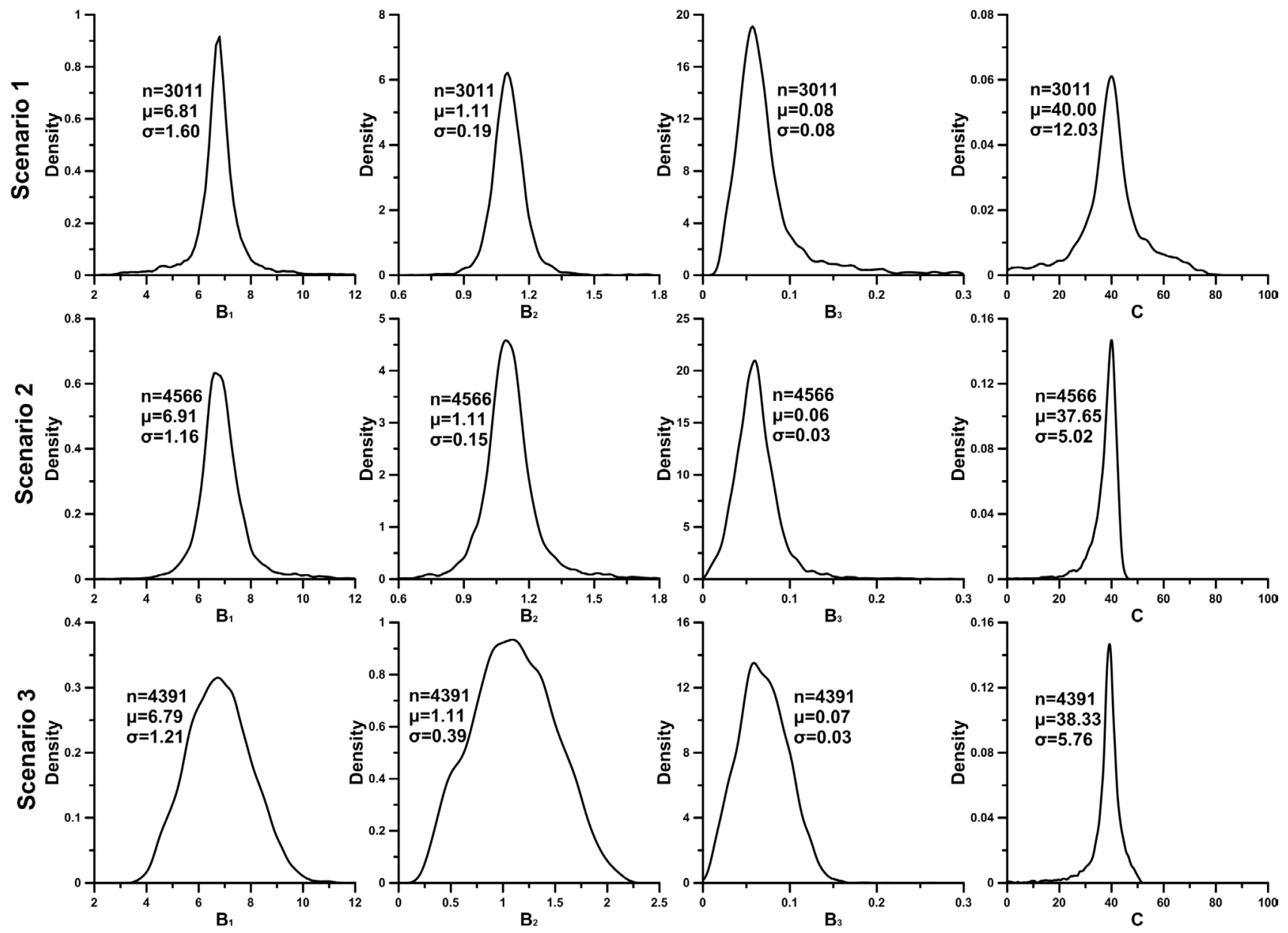

Fig. 2. Distributions of the estimated decay rates $\left(\mathrm{B}_{i}\right)$ and constant ( $\mathrm{C}$, equal to the background intensity) for simulations that have a correctively identified number of components $(\mathrm{k}=3)$ in the three scenarios. $\mathrm{n}$ is the number of decay curves, $\mu$ and $\sigma$ denote the average and standard deviation of a decay rate or a constant, respectively. 
A summary of the estimated numbers of components $(k)$ for the three scenarios is listed in Table 2.

Table 3 shows that the smallest decaying component may not be recognized and that the background is likely to be overestimated if the number of components is underestimated. Fig. 2 shows the distributions of the estimated parameters (decay rates and background constants) with an estimated number of components set to 3 for the three scenarios. Both the estimated decay constants and the backgrounds are comparable to those used for the simulation. Fig. 3 to Fig. 5 show the resulting estimates for the three scenarios.

Scenario 1 results in a success rate (i.e., the number of simulations with the correct number of components, $k=3$ ) of $60.2 \%$ (Table 2). Fig. 3 demonstrates that only a single component can be recognized if few channels ( $\leq 25$ or so) are adopted. Simulations with an identified number of components equal to 2 are likely to fall into two regions: one with fewer than 50 channels and one with a measurement time shorter than $10 \mathrm{~s}$. The estimate is characterized by considerable uncertainty if the number of channels lies between 50 and 100 or if the measurement time lies between 10 and $30 \mathrm{~s}$. This indicates that a longer simulation time or a finer data resolution alone does not necessarily ensure that the estimate is correct. Unsurprisingly, the quality of the estimates improves remarkably with a prolonged measurement time and an increased number of channels. It is interesting to note that the OSL intensity for the smallest decay rate $(0.06)$ decreases to 54.9 and $16.5 \%$ of its initial level at measurement durations of 10 and $30 \mathrm{~s}$, respectively. A short measurement time keeps the OSL intensity from completely depleting, and the influence of this effect would be especially significant for the recognition of slower components. This may also explain why the estimated constants for an estimated number of components equal to 3 or 4 in Fig. 1 (Table 1) using the measured decay curves were significantly larger than the expected back-

Table 2. A summary of the estimated values of $\mathrm{k}$ for the three scenarios. $\mathrm{N}$ is the total numbers of simulations, and Fail denotes the number of failed simulations.

\begin{tabular}{ccccccc}
\hline Scenario & $\boldsymbol{N}$ & Fail & $\boldsymbol{k}=\mathbf{1}$ & $\boldsymbol{k}=\mathbf{2}$ & $\boldsymbol{k}=\mathbf{3}$ & $\boldsymbol{k}=\mathbf{4}$ \\
\hline 1 & 5000 & 1 & 224 & 1763 & 3011 & 1 \\
2 & 5000 & 0 & 1 & 431 & 4566 & 2 \\
3 & 5000 & 0 & 0 & 607 & 4391 & 2 \\
\hline
\end{tabular}

Table 3. Estimated decay rates $\left(\mathrm{B}_{i}\right)$ and constants ( $\mathrm{C}$, equal to the background intensity) for simulations that underestimated the number of components $(\mathrm{k}=2)$ for the three scenarios. Values are mean $\pm S D_{\text {mean. }}$

\begin{tabular}{cccc}
\hline Scenario & $\boldsymbol{B}_{1}$ & $\boldsymbol{B}_{2}$ & $\boldsymbol{C}$ \\
\hline 1 & $4.19 \pm 0.06$ & $0.60 \pm 0.01$ & $60.97 \pm 0.43$ \\
2 & $5.99 \pm 0.06$ & $0.86 \pm 0.01$ & $42.37 \pm 0.09$ \\
3 & $6.45 \pm 0.05$ & $0.94 \pm 0.02$ & $48.69 \pm 0.18$ \\
\hline
\end{tabular}

ground for a reader: if we assume that a bright decay curve contains a slower component (with a decay constant smaller than about 0.06), then it may not be possible for its intensity to decrease to a negligible level under a 40 -s measurement duration.

The results of Scenario 2 (Table 2) show that the proportion of successful estimates was $91.3 \%$ if the decay constants are distinguishable and the data has a moderate resolution. The quality of the estimate is strongly related to the smallest number of trapped electrons (Fig. 4). Many of the estimated numbers of components are 2 $(k=2)$ if the smallest number of trapped electrons is less than 150 or so, and a correct estimate $(k=3)$ is generally obtained when the smallest number of trapped electrons exceeds 500 . If the intensity of a decaying component is not significantly higher than the background level, then it is not likely to be isolated from the curve. Scenario 2 indicates that the BIC is more applicable to decay curves with moderate to high OSL intensities. For dim decay curves of samples with a low signal-to-noise ratio, this approach is likely to underestimate the number of components.

The proportion of successful estimates for Scenario 3 was $87.8 \%$ (Table 2). Fig. 5 shows that the number of components is very likely to be underestimated $(k=2)$ if the smallest decay rate is less than 0.04 or so. Simulations that result in $k=2$ also appear if the difference between the two decay constants is very small (not distinguishable). Under a measurement time of $40 \mathrm{~s}$, the OSL intensity decreases to only $20.2 \%$ of its initial level with a decay constant of 0.04 . The number of correct estimates increases to 4735 (from 4391) for an additional scenario

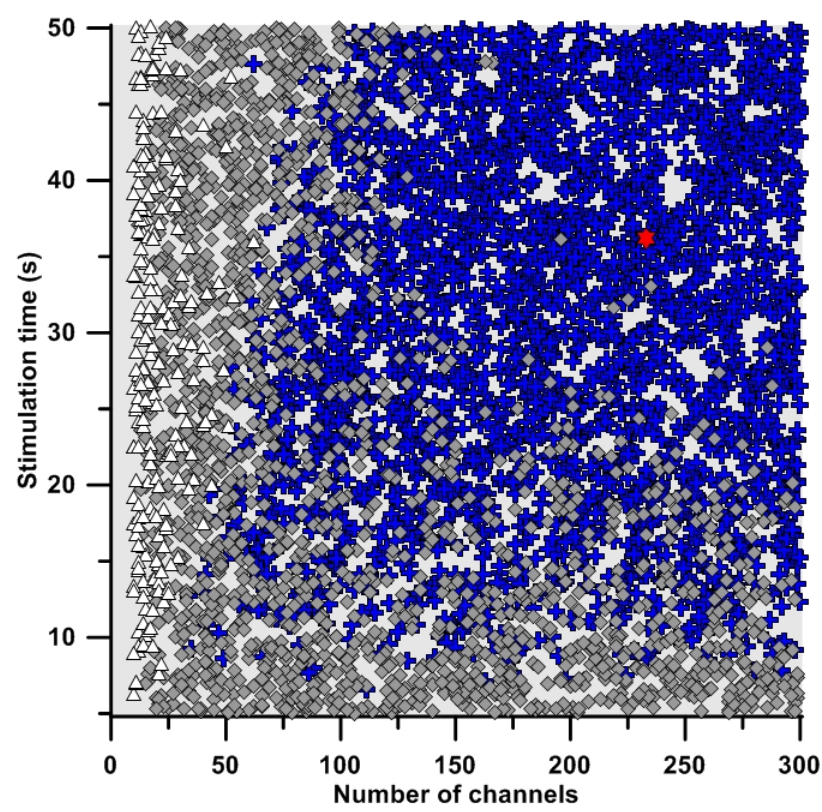

Fig. 3. The estimated number of components (k) for Scenario 1. The white triangles, grey diamonds, blue crosses, and red star represent the results for simulations with $\mathrm{k}=1,2,3$, and 4, respectively. 


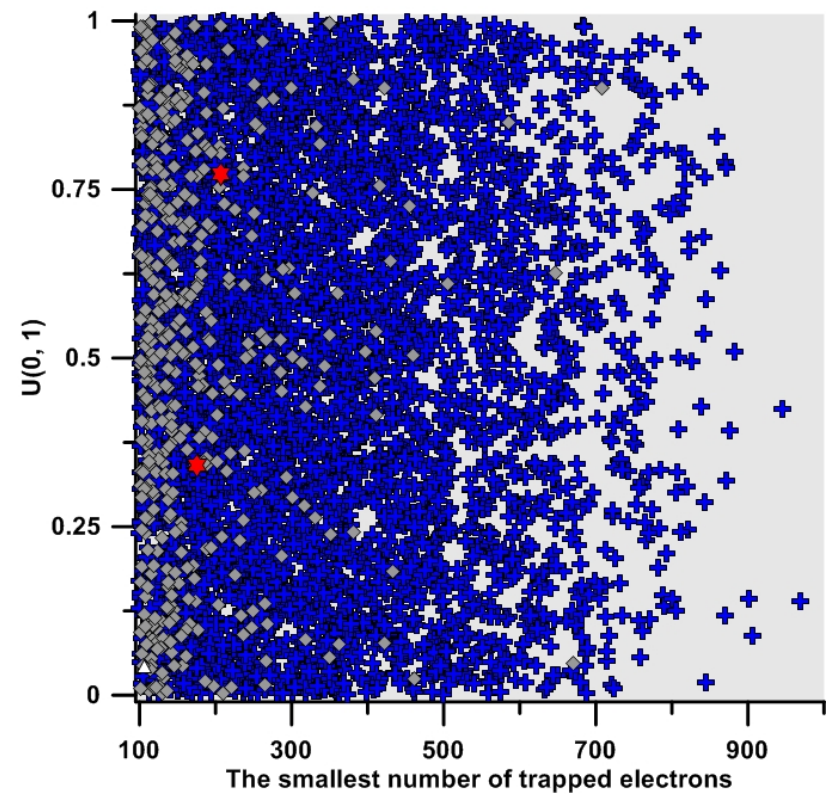

Fig. 4. The estimated number of components (k) for Scenario 2. Values on the $x$-axis are the simulated smallest number of trapped electrons. Values on the $y$-axis are randomized with a uniform generator from the space $(0,1)$. The white triangle, grey diamonds, blue crosses and red stars represent simulations with $\mathrm{k}=1,2,3$, and 4 , respectively.

(not presented) in which the measurement time was set to $80 \mathrm{~s}$. Scenario 3 can be compared with Scenario 1, which has three fixed decay rates but a variable measurement time. Both scenarios suggest that the ability to recognize a slow component correctively from the decay curve depends on whether it is possible for its intensity to decline to a negligible level within the stimulation time. Thus, for a decaying component with a very small decay constant, it may be too time-consuming to allow its intensity to decrease to a negligible level so that it can be isolated.

\section{DISCUSSION AND CONCLUSIONS}

From the three scenarios described in Section 4, it can be concluded that the measurement time, the number of channels, the intensities of the decaying components, and variations of the decay rates can potentially affect the estimation of the optimal number of components using the BIC approach. To decrease the signals of the slower components to negligible levels, it is necessary to use a larger number of channels and a longer stimulation time. A component may not be isolated correctively from the decay curve if its mean life is longer than the stimulation time, as we observed in Scenario 1 and Scenario 3. The problem of an upper limit for the number of channels in the Risø instrument can be solved by conducting a measurement in multiple stages (Adamiec, 2005). The mean life of a decaying component is determined by its decay

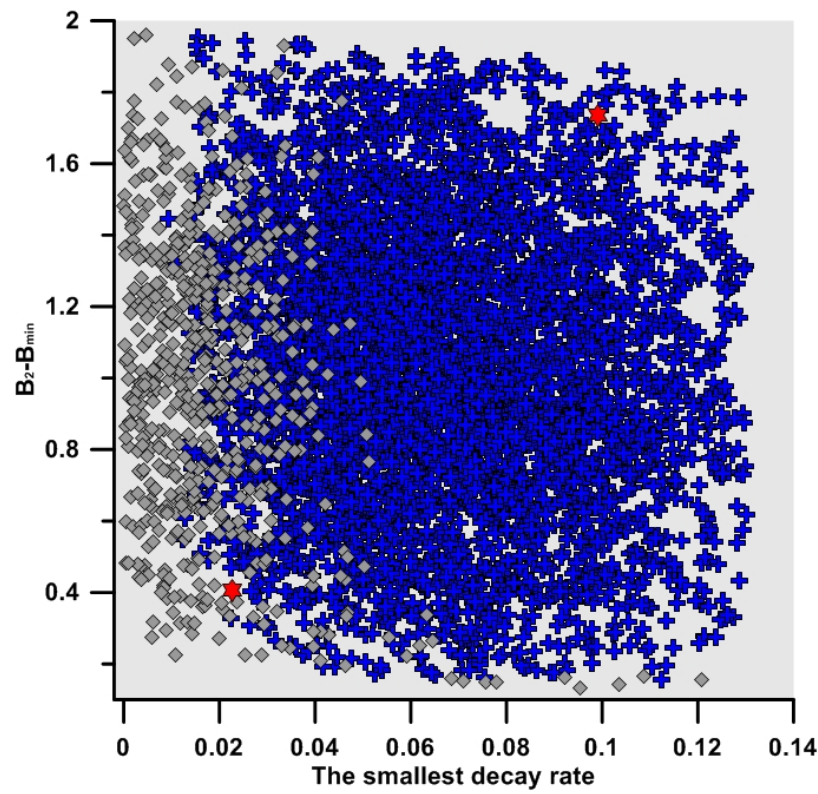

Fig. 5. The estimated number of components (k) for Scenario 3. The smallest decay rate is plotted against the difference between the second-largest decay rate $\left(\mathrm{B}_{2}\right)$ and the smallest decay rate $\left(\mathrm{B}_{\min }\right)$. The grey diamonds, blue crosses and red stars represent simulations with $\mathrm{k}=2,3$ and 4, respectively.

constant. The smallest reported decay constant (S4) is about 0.0003 under stimulation by blue light with a wavelength of $470 \mathrm{~nm}$ (Jain et al., 2003), which means that to identify the component correctly, a very long stimulation time is required. It has been observed that the variation of the decay constants depends on the stimulation wavelength (Singarayer and Bailey, 2003; 2004). Stimulation using light with a shorter wavelength accelerates the decrease of OSL intensity of a decaying component, but the differences between decay constants also decrease. Consequently, it is difficult to achieve reasonable measurement conditions (for the stimulation wavelength, total measurement time, and number of channels) that can both decrease the measurement time and still obtain reliable estimates, particularly since the optimal settings may vary among samples.

The signal-to-noise ratio of a decay curve (or of a decaying component) is determined by its OSL intensity, the background, and the uncertainty of photon counts. The results of Scenario 2 demonstrate that the intensity of a decaying component needs to significantly exceed the background in order to suppress the noise sufficiently to enable correct isolation from the decay curves. Thus, the BIC approach may be more applicable to decay curves that have a moderate to high signal-to-noise ratio. The variation in OSL photon counts are dispersed more than would be expected from the assumed Poisson distribution ( $\mathrm{Li}, 2007)$, and the degree of uncertainty depends on the correction factor (Adamiec et al., 2012). If the correction factor is significantly larger than 1 , then it may 
be more feasible to use a Normal distribution to construct the likelihood function instead of the Poisson distribution used in Eq. 2.3.

In this study, we investigated the potential of using the BIC method to estimate the optimal numbers of components in an OSL decay curve. The simulated scenarios suggest that the quality of the estimation can be affected by several factors. In summary, there are two major limitations for applying the BIC to estimate the optimal number of components for a decay curve: (1) The signal-tonoise ratio of a decaying component may affect the correctness of the component identification, and an incorrect estimate is likely to be obtained for a dim sample; thus, the signal-to-noise ratio should be significantly greater than the background level to avoid this problem. (2) The mean life of a decaying component is determined by the magnitude of its decay constant, and slower components (i.e., with a smaller decay constant) will need longer measurement durations (and possibly more channels) to ensure that the component can be isolated correctly from the decay curve.

\section{ACKNOWLEDGEMENTS}

We thank Andrzej Bluszcz for his kind and helpful guidance during the programming of the differential evolution algorithm. We also thank two anonymous reviewers for their constructive suggestions on earlier versions of this manuscript. This work was funded by the Ministry of Science and Technology of the People's Republic of China (2013CB956000 and 2012CB426501).

\section{REFERENCES}

Adamiec G, 2000. Variations in luminescence properties of single quartz grains and their consequences for equivalent dose estimation. Radiation Measurements 32(5-6): 427-432, DOI 10.1016/S1350-4487(00)00043-3.

Adamiec G, 2005. OSL decay curves-relationship between single- and multiple-grain aliquots. Radiation Measurements 39(1): 63-75, DOI 10.1016/j.radmeas.2004.03.007.

Adamiec G, Heer AJ and Bluszcz A, 2012. Statistics of count numbers from a photomultiplier tube and its implications for error estimation. Radiation Measurements 47(9): 746-751, DOI 10.1016/j.radmeas.2011.12.009.

Bluszcz A and Adamiec G, 2006. Application of differential evolution to fitting OSL decay curves. Radiation Measurements 41(7-8): 886-891, DOI 10.1016/j.radmeas.2006.05.016.

Duller GAT, 2007. Assessing the error on equivalent dose estimates derived from single aliquot regenerative dose measurements. Ancient TL 25(1): 15-24.

Duller GAT, 2008. Single-grain optical dating of Quaternary sediments: why aliquot size matters in luminescence dating. Boreas 37(4): 589-612, DOI 10.1111/j.1502-3885.2008.00051.x.
Duller GAT, Bøtter-Jensen L and Murray AS, 2000. Optical dating of single sand-sized grains of quartz: source of variability. Radiation Measurements 32(5-6): 453-457, DOI 10.1016/S13504487(00)00055-X

Fitzsimmons KE, 2011. An assessment of the luminescence sensitivity of Australian quartz with respect to sediment history. Geochronometria 38(3): 199-208, DOI 10.2478/s13386-011-0030-9.

Galbraith RF, 1988. Graphical display of estimates having differing standard errors. Technometrics 30(3): 271-281, DOI $10.2307 / 1270081$.

Galbraith RF and Roberts RG, 2012. Statistical aspects of equivalent dose and error calculation and display in OSL dating: An overview and some recommendations. Quaternary Geochronology 11: 1-27, DOI 10.1016/j.quageo.2012.04.020.

Galbraith RF, Roberts RG, Laslett GM, Yoshida H and Olley JM, 1999. Optical dating of single grains of quartz from Jinmium rock shelter, northern Australia. Part I: experimental design and statistical models. Archaeometry 41(2): 339-364, DOI 10.1111/j.14754754.1999.tb00987.x.

Jain M, Murray AS and Bøtter-Jensen L, 2003. Characterisation of bluelight stimulated luminescence components in different quartz samples: implications for dose measurement. Radiation Measurements 37(4-5): 441-449, DOI 10.1016/S1350-4487(03)00052-0.

$\mathrm{Li}$ B, 2007. A note on estimating the error when subtracting background counts from weak OSL signals. Ancient TL 25(1): 9-14.

$\mathrm{Li} \mathrm{B}$ and $\mathrm{Li} \mathrm{SH}, 2006 \mathrm{a}$. Comparison of De estimates using the fast component and the medium component of quartz OSL. Radiation Measurements 41(2): $125-136, \quad$ DOI 10.1016/j.radmeas.2005.06.037.

$\mathrm{Li} \mathrm{SH}$ and Li B, 2006b. Dose measurement using the fast component of LM-OSL signals from quartz. Radiation Measurements 41(5): 534-541, DOI 10.1016/j.radmeas.2005.04.029.

Peng J, Dong ZB, Han FQ, Long $\mathrm{H}$ and Liu XJ, 2013. R package numOSL: numeric routines for optically stimulated luminescence dating. Ancient TL 31(2): 41-48. http://CRAN.Rproject.org/package $=$ numOSL.

Peng $\mathrm{J}$ and Han FQ, 2013. Selections of fast-component OSL signal using sediments from the south edge of Tengger Desert. Acta Geoscientica Sinica 34(6): 757-762 (in Chinese with English abstract).

Rhodes EJ, 2007. Quartz single grain OSL sensitivity distributions: implications for multiple grain single aliquot dating. Geochronometria 26: 19-29, DOI 10.2478/v10003-007-0002-5.

Schwarz G, 1978. Estimating the dimension of a model. Annals of statistics 6(2): 461-464, DOI 10.1214/aos/1176344136.

Singarayer JS and Bailey RM, 2003. Further investigations of the quartz optically stimulated luminescence components using linear modulation. Radiation Measurements 37(4-5): 451-458, DOI 10.1016/S1350-4487(03)00062-3.

Singarayer JS and Bailey RM, 2004. Component-resolved bleaching spectra of quartz optically stimulated luminescence: preliminary results and implications for dating. Radiation Measurements 38(1): 111-118, DOI 10.1016/S1350-4487(03)00250-6.

Sivia DS, 1996. Data Analysis: a Bayesian Tutorial. Oxford University Press, Oxford.

Steffen D, Preusser F and Schlunegger F, 2009. OSL quartz age underestimation due to unstable signal components. Quaternary Geochronology 4(5): 353-362, DOI 10.1016/j.quageo.2009.05.015.

Storn R and Price K, 1997. Differential evolution: a simple and efficient adaptive scheme for global optimization over continuous spaces. Journal of Global Optimization 11: 341-359.

Tokuyasu K, Tanaka K, Tsukamoto S and Murray A, 2010. The characteristics of OSL signal from quartz grains extracted from modern sediments in Japan. Geochronometria 37: 13-19, DOI 10.2478/v10003-010-0020-6. 